Revue de recherche interdisciplinaire sur le genre et la sexualité

\title{
Premières socialisations des jeunes lesbiennes et gays en France sous le prisme des rapports de genre
}

Annie Velter et Natacha Chetcuti-Osorovitz

\section{(2) OpenEdition}

1 Journals

Édition électronique

URL : https://journals.openedition.org/sextant/404

DOI : $10.4000 /$ sextant.404

ISSN : 2795-8736

Éditeur

Éditions de l'Université de Bruxelles

Édition imprimée

Date de publication : 1 décembre 2018

Pagination : 131-149

ISBN : 978-2-8004-1636-6

ISSN : $1370-267 X$

\section{Référence électronique}

Annie Velter et Natacha Chetcuti-Osorovitz, «Premières socialisations des jeunes lesbiennes et gays en France sous le prisme des rapports de genre », Sextant [En ligne], 35 | 2018, mis en ligne le 01 novembre 2021, consulté le 08 décembre 2021. URL : http://journals.openedition.org/sextant/404 DOI : https://doi.org/10.4000/sextant.404

\section{(c) (i) (ㅇ)}

La revue Sextant est mise à disposition selon les termes de la Licence Creative Commons Attribution Pas d'Utilisation Commerciale - Partage dans les Mêmes Conditions 4.0 International. 


\title{
Premières socialisations des jeunes lesbiennes et gays en France sous le prisme des rapports de genre
}

\author{
Annie Velter et Natacha Chetcuti-Osorovitz
}

Les mouvements sociaux Lesbiens Gays Bisexuels Transsexuels (LGBT ${ }^{1}$ ), se référant à l'idéal égalitaire, mobilisent à la fois les luttes pour la reconnaissance et l'égalité des personnes LGBT, et la lutte contre le VIH. Depuis plus de trente ans, l'expérience sociale et la construction identitaire de l'homosexualité masculine a été marquée par l'épidémie du viH/sida. Le contexte épidémiologique a évolué ; de maladie létale, le viH/sida est devenu maladie chronique. Bien que les rapports sexuels entre hommes soient le seul mode de transmission du VIH pour lequel le nombre de nouveaux diagnostics ne diminue pas $^{2}$, l'amélioration constante de l'efficacité des traitements antirétroviraux a contribué à un changement dans la perception de la maladie et des comportements sexuels préventifs ${ }^{3}$. Ces changements ont abouti à l'autonomisation des mobilisations homosexuelles masculines quant à la question du

\footnotetext{
${ }^{1}$ Bien qu'en France, les études sur les gays et les lesbiennes, devenues aujourd'hui les études LGBT (pour Lesbiennes, Gay, Bi et Trans*) n'aient pas encore acquis une reconnaissance suffisante en tant que champ spécifique d'études contrairement aux pays anglo-saxons, on utilise plus facilement dans le domaine scientifique les termes gays et lesbiennes pour mettre en valeur les spécificités, en termes de pratiques, d'identités, de modes de vie, de représentations, des cultures gays et lesbiennes, que le terme homosexuel.le qui renvoie davantage à une médicalisation des relations de personnes de même sexe. C'est la raison pour laquelle, nous avons choisi d'utiliser les termes gays et lesbiennes, qui sont aussi entrés dans le langage courant pour évoquer des pratiques homosexuelles, sans impliquer forcément une identification politisée lesbienne ou gay.

${ }^{2}$ F. Cazein, J. Pillonel et al., « Découvertes de séropositivité viH et de sida, France, 2003-2013 », Bulletin épidémiologique hebdomadaire, 9-10, 2015, p. 152-161.

${ }^{3}$ A. Velter, A. Bouyssou-Michel et al., Enquête Presse Gay 2004 Saint-Maurice : Institut de Veille Sanitaire, 2007 (http://opac invssantefr/indexphp?lvl=notice_display\&id=3960).
} 
$\mathrm{VIH}^{4}$, produisant des mutations dans la construction de l'identité collective gay ${ }^{5}$. La lutte pour la reconnaissance et l'égalité des personnes LGBT a, de fait, supplanté la question du VIH. A la fin des années 1990, certaines revendications des mouvements homosexuels aboutissent, signe de la modification du contexte social, comme le montre l'adoption du PACS en France.

Alors que les générations antérieures de lesbiennes et gays ont dû composer avec la contrainte à l'hétérosexualité ${ }^{6}$, on peut s'interroger sur la pérennité de cette contrainte pour les nouvelles générations qui se sont construites dans une médiatisation des discours publics (militants et politiques) concernant la légalisation des unions gays et lesbiennes (1999 pour le PACs et 2013 pour le mariage en France) et des règles de filiation. Comment les normes de genre conditionnent-elles les trajectoires homosexuelles des 18-35 ans dans le contexte contemporain?

Des enquêtes qualitatives ${ }^{7}$ ont montré que l'effet de la contrainte hétérosexuelle et de la sexualité reproductive est plus fort chez les lesbiennes qui présentent davantage de parcours progressifs alliant des partenaires hommes et femmes, contrairement aux gays qui se trouvent dans des parcours plus exclusifs, c'est-à-dire des parcours n'incluant pas des partenaires de sexe différents.

Partant de l'hypothèse que les rapports genrés ${ }^{8}$ influent sur les premières socialisations sexuelles des jeunes gays et lesbiennes, nous utiliserons pour la première fois des données quantitatives pour répondre aux questions suivantes : en quoi les normes de genre influent-elles sur les parcours d'autodéfinition des femmes ayant des rapports sexuels avec des femmes (FSF) et des hommes ayant des rapports sexuels avec des hommes (HSH) ? Comment les normes de genre influencent-elles les socialisations sexuelles et amicales ? Nous entendons ici par socialisation la définition donnée par Muriel Darmon ${ }^{9}$, c'est-à-dire l'ensemble des processus sociaux à travers lesquels les individus deviennent ce qu'ils sont au fur et à mesure de leur parcours. En quoi l'appartenance de classe influe-elle sur les révélations auprès des membres de la famille?

${ }^{4}$ C. Broqua, Agir pour ne pas mourir! Act Up, les homosexuels et le sida, Paris, Sciences Po, 2006.

${ }^{5}$ O. De Busscher, "Saisir l'insaisissable. Les stratégies de prévention du sida auprès des homosexuels et bisexuels masculin en France », in C. Broqua, F. LeRT et Y. Souteyrand (éd.), Homosexualité au temps du sida. Tensions sociales et identitaires, Paris, ANRs-Sciences sociales et Sida, 2003, p. 257-271.

${ }^{6}$ M.-A. Schiltz, « Parcours de jeunes homosexuels dans le contexte du viH : la conquête de modes de vie », Population, 52/6, 1997, p. 1485-1537.

${ }^{7}$ N. Chetcuti-Osorovitz et G. Girard, « L'appropriation d'une sexualité minorisée », revue \& Interrogations ?, 21, décembre 2015, http://www.revue-interrogations.org/Lappropriation-d-une-sexualite (consulté le 6 juillet 2016).

${ }^{8}$ Nous concevons la notion de rapport de genre comme un rapport social structurant la différenciation sociale et hiérarchisée entre femmes et hommes. Cette structure sociale façonne notre perception des attendus du féminin et du masculin et des corps ainsi naturalisés (J. RENNES (éd.), Encyclopédie critique du genre, Paris, La Découverte, 2016).

9 M. Darmon, La socialisation, Paris, Armand Colin, 2007. 
Pour répondre à ces questions, nous nous appuierons sur les données de l'Enquête Presse Gays et Lesbiennes (2011) ${ }^{10}$, première enquête quantitative permettant de décrire, dans une perspective comparative, les socialisations des jeunes lesbiennes et gays aux prismes des rapports de genre et de classes sociales.

\section{Une enquête auprès des minorités sexuelles}

L'Enquête Presse Gay est une enquête transversale auto-administrée anonyme, basée sur le volontariat, dont les premières éditions de 1985 à 2004 s'adressaient exclusivement aux hommes ${ }^{11}$. En 2011, l'enquête a été renouvelée avec, pour la première fois, un volet dédié aux femmes ayant des relations sexuelles avec des femmes (FSF) sous le nom Enquête Presse Gays et Lesbiennes (EPGL ${ }^{12}$ ). Elle a été réalisée selon deux modes de collecte : la presse et Internet. Le questionnaire papier, uniquement à destination des hommes, a été encarté dans un mensuel en juin 2011. Des questionnaires web différents s'adressaient aux hommes et aux femmes; ils ont été mis en ligne du 16 mai au 18 juillet 2011, accessibles à partir d'un site dédié à l'enquête (http://enquêtegayslesbiennes.fr). Soixante et un sites gays ou lesbiens d'information ou de rencontres sexuelles au total ont accepté de relayer l'enquête. Ainsi les internautes, fréquentant ces sites, étaient invités à y participer par le biais de bannières, de messages personnalisés ou encore de recommandations via Facebook.

Au total 13833 questionnaires ont été collectés : 3662 femmes et 10171 hommes ont participé quel que soit le mode de recueil.

\section{Une méthodologie spécifique avec des limites}

A l'instar d'autres études réalisées par Internet, les effectifs importants recrutés par ce mode de recrutement en un laps de temps réduit démontrent son efficacité, tout particulièrement auprès de populations dites difficiles à atteindre, sur des thématiques sensibles ${ }^{13}$. Il convient cependant de souligner certaines limites inhérentes à l'utilisation d'échantillons de convenance. Comme pour la presse écrite, les enquêtes de convenance par Internet sont confrontées à un problème de couverture de la population cible : les personnes ne lisent pas toutes la presse gay et lesbienne ou LGBT,

${ }^{10}$ Nous remercions l'Agence nationale de recherche sur le sida et les hépatites virales (ANRS) pour son soutien scientifique et financier. Nous remercions tous ceux et celles qui ont permis la réalisation et la réussite de cette enquête : les sites internet qui ont publicisé l'enquête, nos collègues de Santé publique France et tous les hommes et femmes qui ont pris le temps de répondre à l'enquête.

11 M. Pollak, Les homosexuels et le SIDA, Sociologie d'une épidémie, Paris, Métailié, 1988 ; M. Pollak et M.-A. Schiltz, « Six années d'enquête sur les homo- et bisexuels masculins face au sida : Livre des données », Bulletin de méthodologie sociologique, 31/1, 1991, p. 32-48 ; A. Velter, A. Bouyssou-Michel et al., Enquête Presse Gay 2004 SaintMaurice, op. cit.

12 A. Velter, L. SABOni et al., « Echantillon de convenance par internet et par la presseEnquête Presse Gays et Lesbiennes 2011 », Bulletin de méthodologie sociologique, 26, 2015, p. 46-66.

13 D. Frippiat et N. Marquis, « Les enquêtes par Internet en sciences sociales : un état des lieux », Population, 65/2, 2010, p. 309-338. 
et ne disposent pas toutes d'un accès à Internet ${ }^{14}$. Par ailleurs, un biais de sélection affecte la constitution d'un échantillon d'enquête de convenance quel que soit le mode de passation. Un travail récent a montré que les individus volontaires pour répondre à des enquêtes de convenance semblaient plus à l'aise avec leur identité sexuelle et mieux intégrés dans la communauté LGBT que les homosexuels interrogés en population générale ${ }^{15}$. A ce stade de l'analyse, nous pouvons également supposer que parmi les participantes FSF des enquêtes en ligne, les jeunes générations utilisent davantage les sites Internet dans les enquêtes en ligne que les générations au-delà de 35 ans, comme le démontre l'âge médian très jeune des répondantes ( 26 ans) ${ }^{16}$. Par ailleurs, il n'existe aucune population de référence LGBT sur laquelle s'appuyer pour redresser ces biais le cas échéant. Ainsi, l'absence de contrôle lors du processus d'inclusion et l'absence de base de sondage ${ }^{17}$ ne permettent pas d'extrapoler les résultats de cette étude à l'ensemble de la population des HSH et des FSF. Malgré ces limites méthodologiques les enquêtes de convenance s'efforcent d'être au plus près des modes de vie, afin de recueillir rapidement les informations les plus pertinentes pour la surveillance des comportements en complément de la surveillance épidémiologique des infections sexuellement transmissibles dont le VIH. Mais si l'un des objectifs de ces enquêtes est bel et bien de suivre l'évolution des comportements sexuels préventifs des HSH, elles donnent aussi à voir les évolutions des modes de vie. Les lesbiennes peu ciblées sont de fait peu participantes, parce qu'à tort ou à raison, elles se sentent peu concernées. Au-delà de ces aspects, l'importance des effectifs recrutés assure, depuis plusieurs décennies, la « robustesse des conclusions » de ces enquêtes et permettent de procéder à des analyses fines de comportements spécifiques, ou encore à des comparaisons de sous-groupes ${ }^{18}$ comme nous le proposons dans cette étude.

Le questionnaire adressé aux hommes est, comme les éditions antérieures, centré sur les risques induits par le VIH/sida, interrogeant à la fois les modes de vie et l'activité sexuelle afin d'établir une typologie des stratégies mises en œuvre face au risque de contamination par le vIH/sida. Le questionnaire proposé aux femmes est centré aussi sur la sexualité des lesbiennes par la description des pratiques sexuelles et la santé sexuelle (suivi gynécologique, dépistage des infections sexuellement transmissibles). Les deux questionnaires recueillent d'autres caractéristiques liées à la santé : la consommation de produits psychoactifs ou la santé mentale. Malgré

14 Ibid.

15 P. Prah, F. Hickson et al., « Men who have sex with men in Great Britain : comparing methods and estimates from probability and convenience sample surveys », Sexually Transmitted Infections, 92/6, 2016, p. 455-463.

16 CREDOC, 2010, http://www.credoc.fr/pdf/Rech/C273.pdf, p. 32 et 33 (consulté le 14 novembre 2016). Voir aussi : N. Chetcuti-Osorovitz, " Sexualités entre femmes et usage numérique », Sociologia Historica, 6, 2016, p. 127-152, http://revistas.um.es/sh/article/ view/278681 (consulté le 4 février 2017) ; ID., « Auto-nomination lesbienne avec les réseaux numériques », Hermès, 69, 2014, p. 39-41.

17 C. Beyer, S. Baral et al., « Global Epidemiology of Hiv Infection in Men Who Have Sex with Men », Lancet, 380/9839, 2012, p. 367-377.

18 M.-A. SchiLtz, Les homosexuels face au sida : enquête 1995. Regard sur une décennie d'enquêtes, Rapport CAMs/Cermes/ANRs, 1998. 
ces spécificités genrées, les variables décrivant les profils socio-démographiques, les trajectoires sexuelles et les pratiques de socialisation sont similaires dans les deux questionnaires (encadré 1).

\section{Encadré 1. Variables utilisées}

Des données quantitatives originales sur les trajectoires homosexuelles

Les processus sociaux par lesquels les répondant.e.s se sont construit.e.s ont été analysés à partir de plusieurs questions. Des indicateurs sur le milieu d'appartenance sociale du et de la répondante ont été définis à partir de trois variables : le niveau scolaire regroupé en 4 catégories (inférieur au baccalauréat - études primaires, collèges, lycée ; baccalauréat ; $1^{\text {er }}$ cycle universitaire, BTS, DUT ; $2^{\mathrm{e}}$ ou $3^{e}$ cycle universitaire et grandes écoles) ; les professions et catégories sociales (PCS) regroupées en 6 catégories (agriculteurs, artisans/commerçants/chefs d'entreprise de 10 salariés ou plus, cadres et professions intellectuelles supérieures, professions intermédiaires/techniciens/contremaîtres, employés, ouvriers) et enfin le capital économique catégorisé à partir des réponses à la question sur le montant du revenu net mensuel des répondants (faible : moins de 600 à 999 euros ; moyen : de 1000 à 2299 euros ; aisé : de 2300 à plus de 3000 euros). Un second indicateur sur le milieu social d'origine a été construit en s'appuyant sur la PCs du père et de la mère des répondant.e.s.

Les éléments de la biographie sexuelle ont été recueillis de manière similaire pour les FSF et les HSH : l'âge au premier rapport sexuel, le sexe du partenaire et son âge, le nombre de partenaires de même sexe ou pas au cours de la vie et dans les douze derniers mois.

Les actions socialisatrices sont appréhendées à partir de questions portant sur la participation à des associations politiques ou sportives ; la fréquentation d'espaces homosexuels que ce soient des établissements comme des bars, des établissements où les échanges sexuels sont possibles (tels les saunas ou backrooms), des sites Internet ou la presse gay et lesbienne et LGBT et enfin le réseau amical.

Nous avons restreint notre corpus d'étude, aux répondants.es âgés.es de 15 à 35 ans, pour plusieurs raisons. D'une part, une première comparaison de la structure par âge des lesbiennes et des gays de l'enquête met en lumière des disparités importantes. Ainsi, les 3662 répondantes sont âgées en médiane de 26 ans (IQ : 14-71) alors que les 10171 répondants sont âgés en médiane de 35 ans (IQ : 14-89). Ces âges sont en adéquation avec les intérêts susceptibles d'inciter les lesbiennes et les gays à répondre à ce type d'enquête fondée sur le volontariat. Le très jeune âge des répondantes est caractéristique des femmes qui répondent à ce type d'enquête sur Internet ${ }^{19}$, qui utilisent la toile numérique, les blogs, les revues et les sites d'informations LGBT ${ }^{20}$. Quant à l'âge médian des répondants, il correspond à un moment de la vie où l'activité sexuelle est soutenue et où la question du VIH et des autres IST reste prégnante dans une population où la probabilité d'être contaminé par le VIH est 200 fois plus importante que parmi les hommes hétérosexuels ${ }^{21}$. Aussi, afin de réduire les effets d'âge dans les comparaisons, la population d'étude de cet article est restreinte aux répondantes et

19 V. SCHICK, J.-G. Rosenberger et al., « Sexual behaviour and risk reduction strategies among a multinational sample of women who have sex with women », Sexually Transmitted Infections, 88, 2012, p. 407-412.

${ }^{20}$ N. Chetcuti-Osorovitz, « Sexualités entre femmes et usage numérique », op. cit. ; ID., «Auto-nomination lesbienne avec les réseaux numériques », op. cit.

21 S. Le Vu, Y. Le Strat et al., « Population-based Hiv-1 Incidence in France, 2003-08 : A Modelling Analysis », Lancet Infectious Diseases, 11/3, 2010, p. 682-687. 
répondants âgés de 15 à 35 ans, nés après 1976, soit au final 2684 femmes et 5109 hommes.

D'autre part, bien que le découpage soit peu habituel, la classe d'âge - 15-35 ans - correspond à un cycle de vie commun aux trajectoires homosexuelles : entrée dans la sexualité, expériences diversifiées de partenaires et recherche d'une définition de soi, même si dans le groupe d'âge des plus jeunes il est probable que l'on sélectionne des personnes sexuellement précoces et plus affirmées quant à leur identité que leurs aînées ${ }^{22}$. Cette classe d'âge correspond à la succession d'événements personnels, le temps de la fin des études, c'est aussi l'âge des " choix » progressifs en termes de sexualité et d'auto-nomination. Après 35 ans, c'est davantage la période de stabilisation et de confirmation des " choix » sexuels et conjugaux ${ }^{23}$. Ces âges peuvent être une période de reconfiguration après une séparation, un divorce après donc une période de vie hétérosexuelle. Ces événements peuvent faire suite à une affirmation, dans un processus d'acceptation de son orientation sexuelle dans une mise à distance de la norme hétérosexuelle. Cette observation était déjà présente, pour les hommes, dans les éditions antérieures de l'enquête Presse Gay : Marie-Ange Schiltz y remarquait qu' « au fil des ans, une plus forte proportion de répondants se fixe dans un choix homosexuel $»^{24}$.

\section{Des profils socio-démographiques marqués par des inégalités sociales de genre}

L'analyse des caractéristiques socio-démographiques de la population étudiée montre que les FSF et les HSH ayant participé à cette enquête sont très diplômés, appartiennent à des catégories socio-professionnelles privilégiées et résident en zone urbaine comme le décrivaient les éditions antérieures ${ }^{25}$. Cependant, des distorsions de genre sont observées. En termes d'âge, malgré la restriction de l'échantillon aux participant.e.s âgé.e.s de 15 à 35 ans, les répondantes sont plus jeunes que les répondants : $26 \%$ d'entre elles sont âgées de 15 à 20 ans contre $16 \%$ des hommes. Cet effet d'âge influe sur le statut professionnel. Ainsi les répondantes sont pour près de la moitié d'entre elles étudiantes, alors que ce n'est le cas que pour un tiers des répondants. De même, les répondantes sont moins diplômées des $2^{\mathrm{e}}$ et $3^{\mathrm{e}}$ cycles universitaires et des grandes écoles que les répondants ( $43 \%$ vs $46 \%)$. Cependant, après stratification sur l'âge, les lesbiennes sont aussi diplômées que les gays à l'exception de la tranche d'âge 26-30 ans où les FSF sont 59\% à avoir un diplôme de $2^{\mathrm{e}}$ cycle universitaire et plus contre $55 \%$ pour leur homologue masculin $(\mathrm{p}<0,000)$. Et pour autant, les FSF

22 N. Methy, A. Velter, C. Semaille, N. Bajos, « Sexual behaviours of homosexual and bisexual men in France : a generational approach », PloS One, 10/3:e0123151, 2015, doi : 10.1371/journal.pone.0123151 PMID: 25816322.

23 N. Chetcuti-Osorovitz et G. Girard., «L'appropriation d'une sexualité minorisée », op. cit. ; N. Chetcuti, Se dire lesbienne, vie de couple, représentation de soi, Paris, Payot, 2013 [2010].

${ }^{24}$ M.-A. Schiltz, « Parcours de jeunes homosexuels dans le contexte du VIH », op. cit.

25 M.-A. Schiltz, Les homosexuels face au sida : enquête 1995. Regard sur une décennie d'enquêtes, op. cit ; A. Velter, A. Bouyssou-Michel et al., Enquête Presse Gay 2004 SaintMaurice, op. cit. 
actives occupent moins souvent des postes de cadres et professions intellectuelles supérieures que les HSH (33\% vs 40\%) et ce, quel que soit leur âge. Elles exercent plus souvent des professions intermédiaires ( $26 \%$ vs $21 \%$ ) ou sont plus souvent employées ( $31 \%$ vs $27 \%$ ). Ces distinctions restent vraies voire s'accentuent pour les diplômé.e.s de $2^{\mathrm{e}}$ et $3^{\mathrm{e}}$ cycle universitaire et des grandes écoles : $57 \%$ de femmes très diplômées sont cadres ou exercent des professions intellectuelles supérieures contre $67 \%$ pour les hommes ( $\mathrm{p}<0,000), 25 \%$ d'entre elles exercent une profession intermédiaire pour $17 \%$ des hommes et $15 \%$ sont employées contre $12 \%$ des hommes $(\mathrm{p}<0,000)$.

De manière cohérente avec les inégalités de genre portant sur le rapport entre l'emploi, le sexe, le secteur d'activité et le revenu ${ }^{26}$ dans la population hétérosexuelle, ces disparités se retrouvent dans la population homosexuelle : les lesbiennes ont un capital économique moindre que les gays. Alors que 53\% d'entre elles ont déclaré un niveau de revenu faible contre $37 \%$ pour les gays, elles ne sont que $5 \%$ à déclarer un niveau de revenu aisé contre $15 \%$ pour les gays $(\mathrm{p}<0,000)$. Ces différences se vérifient quel que soit l'âge des participant.e.s, le niveau de diplôme ou encore la profession exercée.

Les lesbiennes appartiennent plus souvent que les gays à un milieu d'origine favorisé : $48 \%$ sont dans ce cas contre $41 \%(p<0,000)$, et ce quel que soit leur âge, bien que les écarts tendent à diminuer avec l'âge et ne soient plus significatifs.

Quel que soit leur sexe, les participant.e.s résident plus souvent dans des zones urbaines. Cette surreprésentation des urbains a été observée de manière récurrente par de nombreux travaux menés auprès des homosexuels ${ }^{27}$, rendant compte de l'attractivité des centres urbains où l'homosexualité peut être plus facilement vécue. Avec l'âge, de manière similaire pour les FSF et les HSH, on observe une mobilité géographique des petites villes vers les grandes agglomérations.

${ }^{26}$ Insee, 2014, http://www.insee.fr/fr/themes/tableau.asp?reg_id=0\&ref_id=NATCCF03172 (consulté le 6 juillet 2016).

${ }^{27}$ M. Pollak, M.-A. Schiltz, "Identité sociale et gestion d'un risque de santé », Actes de la recherche en sciences sociales, 68, 1987, p. 77-102 ; A. Messiah, E. Mouret-Fourme, «Homosexualité, bisexualité : éléments de sociobiographie sexuelle », Population, 48/5, 1993, p. 1353-1379 ; M.-A. Schiltz, « Parcours de jeunes homosexuels dans le contexte du VIH », op. cit.; W. Rault, « Les mobilités sociales géographiques des gays et des lesbiennes », Sociologie, 4/7, 2016, http://sociologie.revues.org/2894 (consulté le 23 septembre 2017). 
Tableau 1. Caractéristiques sociales des lesbiennes et des gays ayant répondu à l'EPGL 2011 âgés de 15 à 35 ans

\begin{tabular}{|c|c|c|c|c|c|c|c|}
\hline & \multicolumn{2}{|c|}{ Femmes } & \multicolumn{2}{|c|}{ Hommes } & \multicolumn{2}{|c|}{ Total } & \multirow{2}{*}{\begin{tabular}{|l} 
Ecart F-H \\
$p$-value
\end{tabular}} \\
\hline & Effectifs & $\%$ & Effectifs & $\%$ & Effectifs & $\%$ & \\
\hline \multicolumn{8}{|l|}{ Age à l'enquête (15-35) } \\
\hline $15-20$ ans & 661 & 24,6 & 836 & 16,3 & 1497 & 19,2 & 0,000 \\
\hline $21-25$ ans & 988 & 36,8 & 1604 & 31,4 & 2592 & 33,3 & \\
\hline $26-30$ ans & 623 & 23,2 & 1388 & 27,2 & 2011 & 25,8 & \\
\hline $31-35$ ans & 412 & 15,4 & 1281 & 25,1 & 1693 & 21,7 & \\
\hline Total & 2684 & 100,0 & 5109 & 100,0 & 7793 & 100,0 & \\
\hline Age médian & \multicolumn{2}{|c|}{24 [21-28] } & \multicolumn{2}{|c|}{$26[22-31]$} & & & \\
\hline Niveau d'études & & & & & & & 0,062 \\
\hline Inferieur au bac & 417 & 15,7 & 772 & 15,3 & 1189 & 15,4 & \\
\hline $\mathrm{Bac}$ & 383 & 14,4 & 677 & 13,4 & 1060 & 13,7 & \\
\hline $1^{\mathrm{er}}$ cycle universitaire, BTS, DUT & 711 & 26,7 & 1269 & 25,0 & 1980 & 25,7 & \\
\hline $\begin{array}{l}2^{\mathrm{e}} \text { ou } 3^{\mathrm{e}} \text { cycle universitaire, } \\
\text { grandes écoles }\end{array}$ & 1147 & 43,2 & 2344 & 46,3 & 3491 & 45,2 & \\
\hline Total & 2658 & 100,0 & 5062 & 100,0 & 7720 & 100,0 & \\
\hline Statut professionnel & & & & & & & 0,000 \\
\hline Etudiant & 1295 & 48,3 & 1735 & 34,0 & 3030 & 38,9 & \\
\hline Salarié/indépendant & 1148 & 42,8 & 2924 & 57,3 & 4072 & 52,3 & \\
\hline Chômeur & 170 & 6,3 & 342 & 6,6 & 512 & 6,6 & \\
\hline Retraité/autre inactif & 70 & 2,6 & 105 & 2,1 & 175 & 2,2 & \\
\hline Total & 2683 & 100,0 & 5106 & 100,0 & 7789 & 100,0 & \\
\hline Emploi (csp 2003) & & & & & & & 0,000 \\
\hline Agriculteurs & 5 & 0,4 & 12 & 0,3 & 17 & 0,3 & \\
\hline $\begin{array}{l}\text { Artisans/commerçants/chefs d'en- } \\
\text { treprise de } 10 \text { salariés ou plus }\end{array}$ & 55 & 3,9 & 166 & 4,9 & 221 & 4,6 & \\
\hline $\begin{array}{l}\text { Cadre et professions } \\
\text { intellectuelles supérieures }\end{array}$ & 479 & 33,4 & 1369 & 40,2 & 1848 & 38,2 & \\
\hline $\begin{array}{l}\text { Professions intermédiaires / } \\
\text { techniciens/contremaîtres }\end{array}$ & 367 & 25,6 & 711 & 20,9 & 1078 & 22,3 & \\
\hline Employés & 452 & 31,5 & 926 & 27,2 & 1378 & 28,5 & \\
\hline Ouvriers & 75 & 5,2 & 221 & 6,5 & 296 & 6,1 & \\
\hline Total & 1433 & 100,0 & 3405 & 100,0 & 4838 & 100,0 & \\
\hline Capital économique Ego & & & & & & & 0,000 \\
\hline Faible & 1302 & 53,5 & 1827 & 37,9 & 3129 & 43,1 & \\
\hline Moyen & 1008 & 41,4 & 2277 & 47,2 & 3285 & 45,3 & \\
\hline
\end{tabular}




\begin{tabular}{|c|c|c|c|c|c|c|c|}
\hline Aisé & 123 & 5,1 & 717 & 14,9 & 840 & 11,6 & \\
\hline Total & 2433 & 100,0 & 4821 & 100,0 & 7254 & 100,0 & \\
\hline Milieu social-csP EGo & & & & & & & 0,000 \\
\hline Favorisé & 479 & 33,4 & 1369 & 40,2 & 1848 & 38,2 & \\
\hline Intermédiaire & 427 & 29,8 & 889 & 26,1 & 1316 & 27,2 & \\
\hline Populaire & 527 & 36,8 & 1146 & 33,7 & 1673 & 34,6 & \\
\hline Total & 1433 & 100,0 & 3404 & 100,0 & 4837 & 100,0 & \\
\hline \multicolumn{7}{|l|}{ Milieu social-csp d'origine (père-mère) } & 0,000 \\
\hline Favorisé & 1281 & 48,4 & 2051 & 41,1 & 3332 & 43,7 & \\
\hline Intermédiaire & 814 & 30,8 & 1674 & 33,6 & 2488 & 32,6 & \\
\hline Populaire & 549 & 20,8 & 1261 & 25,3 & 1810 & 23,7 & \\
\hline Total & 2644 & 100,0 & 4986 & 100,0 & 7630 & 100,0 & \\
\hline Résider & & & & & & & 0,000 \\
\hline Seul.e & 716 & 26,7 & 2137 & 41,9 & 2853 & 36,6 & \\
\hline $\begin{array}{l}\text { En couple avec une personne de } \\
\text { même sexe }\end{array}$ & 88 & 3,3 & 1237 & 24,2 & 1325 & 17,1 & \\
\hline Avec son père et / ou sa mère & 749 & 27,9 & 1136 & 22,2 & 1885 & 24,2 & \\
\hline $\begin{array}{l}\text { Autre (co-location, un membre de } \\
\text { sa famille, ...) }\end{array}$ & 1128 & 42,1 & 595 & 11,7 & 1723 & 22,1 & \\
\hline Total & 2681 & 100,0 & 5105 & 100,0 & 7786 & 100,0 & \\
\hline \multicolumn{3}{|c|}{ Taille d'agglomération en 3 classes (100 000) } & & & & & 0,005 \\
\hline$<20000$ habitants & 841 & 31,4 & 1476 & 28,9 & 2317 & 29,8 & \\
\hline 20000 à 99999 habitants & 575 & 21,4 & 1022 & 20,0 & 1597 & 20,5 & \\
\hline 100000 habitants et + & 1266 & 47,2 & 2608 & 51,1 & 3874 & 49,7 & \\
\hline Total & 2682 & 100,0 & 5106 & 100,0 & 7788 & 100,0 & \\
\hline
\end{tabular}

Lecture : $48,3 \%$ des lesbiennes et $34,0 \%$ des gays âgés de 15 à 35 ans ont déclaré être étudiants.

Source : Enquête Presse Gays et Lesbiennes 2011.

\section{Une contrainte à l'hétérosexualité plus forte pour les lesbiennes que pour les gays}

\section{Affirmation identitaire et variabilités selon les positions de genre,} de statut social et de niveaux d'études

Prendre en compte la question de l'autodéfinition de l'orientation sexuelle permet de mettre en regard l'autodésignation et les pratiques sexuelles des répondant.e.s et de mieux cerner la place qu'ils/elles accordent à leurs relations tant homosexuelles qu'hétérosexuelles ${ }^{28}$.

${ }^{28}$ B. Lhomond et S. Michaels, « Homosexualité/hétérosexualité : les enquêtes sur les comportements sexuels en France et aux USA ", Journal des anthropologues, 82-83, 2000, p. 91-111. 
Globalement, une très large majorité des répondant.e.s se définit comme homosexuelle : $86 \%$ pour les HSH et $71 \%$ pour les FSF, confirmant la sélection des personnes qui répondent à ce type d'enquêtes ${ }^{29}$. La participation fondée sur le volontariat explique cette sélection des répondant.e.s nécessitant une affirmation de son identité sexuelle.

Des disparités sont observées entre les FSF et les HSH. L'identification est plus diversifiée, moins affirmée chez les FSF que chez les HSH. Les FSF s'autodésignent homosexuelles moins souvent que les HSH et ce, quels que soient leur âge, leur appartenance sociale, leur capital socio-culturel ou la taille de l'agglomération dans laquelle elles résident.

D'une manière générale, la bisexualité est plus souvent rapportée par les FSF que par les HSH. A 15-20 ans, les répondantes sont 26\% à s'identifier comme bisexuelles contre $16 \%$ pour les répondants ; respectivement à $31-35$ ans, elles sont $10 \%$ contre $6 \%$ chez leurs homologues masculins. Par rapport à leurs aînés, les jeunes des deux sexes revendiquent une bisexualité illustrant l'ambivalence et la difficulté d'affirmer des préférences sexuelles en début de carrière sexuelle. Ce moment de «nomadisme» identitaire a été décrit auparavant pour les $\mathrm{HSH}^{30}$. Ainsi en 1997, 18\% des répondants à l'EPG âgés de 16 à 20 ans se définissaient comme bisexuels ${ }^{31}$. Cette proportion, peu différente de celle d'aujourd'hui, rend compte du caractère inexorable de cette période transitoire, quelle que soit la génération.

Pour autant, le mode de recrutement par Internet permet d'atteindre des hommes situés plus à la marge de la « communauté gay », la proportion d'HSH qui se définissent comme bisexuels étant en augmentation par rapport aux éditions antérieures ${ }^{32}$.

Plus les répondant.e.s avancent en âge, plus leur capital socio-culturel est élevé, plus leur résidence est urbaine et plus ils/elles s'identifient comme homosexuel.le.s. Les tendances sont similaires pour les FSF et les HSH.

\section{Trajectoires sexuelles et spécificités gays et lesbiennes}

Le sexe des partenaires au cours de la vie et dans les douze derniers mois rend compte des spécificités des parcours de vie des lesbiennes et des gays et des rapports sociaux de sexe. La sexualité au cours de la vie des FSF de notre étude est marquée par une bisexualité importante : la moitié d'entre elles ont eu des rapports sexuels avec des femmes et des hommes contre $30 \%$ des participants. De même, $6 \%$ des femmes rapportent des rapports exclusivement hétérosexuels sur les douze derniers mois, alors qu'aucun des répondants n'a eu de rapports sexuels avec des femmes. Les répondantes sont un tiers à avoir eu des rapports sexuels uniquement avec des personnes de même sexe contre deux tiers pour les répondants. Le sexe du premier partenaire sexuel et l'âge médian au premier rapport traduisent aussi cette pression à

${ }^{29}$ A. Velter, L. SAboni et al., « Echantillon de convenance par internet et par la presse », op. cit.

30 A. Messiah et E. Mouret-Fourme, « Sexualité et sciences sociales : les apports d'une enquête », Population, 48/5, 1993, p. 1353-1379.

31 M.-A. Schiltz., « Parcours de jeunes homosexuels dans le contexte du VIH », op. cit.

${ }^{32}$ A. Velter, L. SAboni et al., « Echantillon de convenance par internet et par la presse », op. cit. 
l'hétéronormalité des femmes par rapport aux hommes. Les répondants sont $82 \%$ à déclarer avoir eu leur premier rapport sexuel avec une personne du même sexe contre $48 \%$ des répondantes $(\mathrm{p}<0,0001)$. Aussi l'âge médian au premier rapport sexuel avec une personne de même sexe pour les répondantes est plus élevé que l'âge médian avec une personne de sexe différent contrairement aux répondants dont l'âge médian au premier rapport n'est pas différent quel que soit le sexe du premier partenaire sexuel.

A l'instar de la différence entre les déclarations des femmes et des hommes hétérosexuels sur le nombre de partenaires au cours de la vie ${ }^{33}$, les FSF rapportent un nombre de partenaires plus faible que les hommes quel que soit le sexe des partenaires : le nombre médian de partenaires féminines pour les femmes est de 3 contre 20 partenaires masculins pour les hommes. Ces différences sont observées à tous les âges. Cet écart met en lumière les différences d'appréciations des relations en référence aux stéréotypes de genre associant le féminin aux sentiments et le masculin à la performance sexuelle ${ }^{34}$. Les normes sociales qui prescrivent d'établir un lien entre sexualité et affectivité, plus intériorisées par les femmes ${ }^{35}$ sont aussi intériorisées par les FSF et les HSH. Par rapport aux HSH, les FSF rapportent plus souvent avoir une relation stable avec une personne du même sexe (70\% vs 61\%) et sont d'ailleurs plus souvent PACsées (14\% vs 10\%). La sexualité des FSF s'exprime dans un cadre relationnel de proximité affective, indispensable comme celle des femmes hétérosexuelles ${ }^{36}$.

A l'instar des données des enquêtes en population générale, les femmes qui ont des rapports sexuels avec des femmes, ou des femmes avec des hommes, ont des trajectoires plus diversifiées du point de vue du sexe des partenaires que les HSH $^{37}$. Cette pression sociale à l'hétérosexualité s'illustre par la réalisation d'un parcours plus rapidement exclusif pour les gays que pour les lesbiennes ${ }^{38}$. En outre, le phénomène d'invisibilité du lesbianisme joue un rôle majeur dans l'initiation à la sexualité qui n'outrepasse pas les effets générationnels ${ }^{39}$; ce qui explique que dans le type d'échantillon présenté dans notre étude, on observe un début de parcours pour les FSF plus souvent marqué par des pratiques bisexuelles que pour les HSH. Pour autant, nos données transversales ne permettent pas de présupposer que les préférences sexuelles tendraient à se spécialiser au cours de la « carrière sexuelle » des FSF. On peut supposer aussi que la diversité des trajectoires sexuelles et la spécificité des parcours de vie

${ }^{33}$ M. Bozon, « Premier rapport sexuel, première relation : des passages attendus ", in N. Bajos et M. Bozon (éd.), Enquête sur la sexualité en France. Pratiques, genre et santé, Paris, La Découverte, 2008, p. 117-147.

${ }^{34}$ F. Maillochon, « Dire et faire : évolution des normes de comportements sexuels des jeunes dans la seconde partie du $\mathrm{Xx}^{\mathrm{e}}$ siècle », in G. PAicheler et M.-A. Loyola (éd.), Sexualités, normes et contrôle social, Paris, L'Harmattan, 2003, p. 117-132.

${ }^{35} \mathrm{M}$. Bozon, « Premier rapport sexuel, première relation », op. cit.

${ }^{36}$ F. MAILlochon, « Dire et faire », op. cit.

${ }^{37}$ N. Chetcuti, N. Beltzer et al., « Preventive care's forgotten women: life course, sexuality, and sexual health among homosexually and bisexually active women in France », The Journal of Sex Research, 50/6, 2013, p. 587-597.

${ }^{38}$ N. Chetcuti-Osorovitz et G. Girard., «L'appropriation d'une sexualité minorisée », op. cit. ; N. Chetcuti, Se dire lesbienne, vie de couple, représentation de soi, op. cit.

${ }^{39}$ Ibid. 
empruntés par des personnes ayant des rapports homosexuels, peut se lire comme une réponse à un contexte social qui peine à dépasser, au-delà des avancées des politiques de lutte contre les discriminations LGBT, une acceptation de principe de l'homosexualité ${ }^{40}$.

Tableau 2. Trajectoires sexuelles des lesbiennes et des gays ayant répondu à l'EPGL 2011 âgés de 15 à 35 ans

\begin{tabular}{|c|c|c|c|c|c|c|c|}
\hline & \multicolumn{2}{|c|}{ Femmes } & \multicolumn{2}{|c|}{ Hommes } & \multicolumn{2}{|c|}{ Total } & \multirow{2}{*}{$\frac{\text { Ecart F-H }}{p \text {-value }}$} \\
\hline & Effectifs & $\%$ & Effectifs & $\%$ & Effectifs & $\%$ & \\
\hline \multicolumn{8}{|l|}{ Nomination de soi } \\
\hline Homosexuel.le & 1891 & 70,7 & 4404 & 86,4 & 6295 & 81,0 & 0,000 \\
\hline Bisexuel.le & 447 & 16,7 & 511 & 10,0 & 958 & 12,3 & \\
\hline Hétérosexuel.le & 98 & 3,7 & 19 & 0,4 & 117 & 1,5 & \\
\hline Autre & 29 & 1,1 & 13 & 0,3 & 42 & 0,5 & \\
\hline Vous refusez de vous définir & 209 & 7,8 & 150 & 2,9 & 359 & 4,6 & \\
\hline Total & 2674 & 100,0 & 5097 & 100,0 & 7771 & 100,0 & \\
\hline Sexualité au cours de la vie & & & & & & & 0,000 \\
\hline Femmes uniquement & 879 & 32,8 & 0 & 0 & 879 & 11,3 & \\
\hline Hommes uniquement & 156 & 5,8 & 3360 & 65,8 & 3516 & 45,1 & \\
\hline Femmes et hommes & 1420 & 52,9 & 1510 & 29,6 & 2930 & 37,6 & \\
\hline Non & 229 & 8,5 & 239 & 4,6 & 468 & 6,0 & \\
\hline Total & 2684 & 100,0 & 5109 & 100,0 & 7793 & 100,0 & \\
\hline \multicolumn{8}{|c|}{ Age médian premier rapport sexuel } \\
\hline Avec une femme & \multicolumn{2}{|c|}{19 [17-21] } & \multicolumn{2}{|c|}{$17[16-19]$} & & & \\
\hline Avec un homme & \multicolumn{2}{|c|}{$17[15-18]$} & \multicolumn{2}{|c|}{$17[15-18]$} & & & \\
\hline \multicolumn{8}{|c|}{ Nombre médian de partenaires - vie } \\
\hline Femmes & \multicolumn{2}{|c|}{$3[1-6]$} & \multicolumn{2}{|c|}{$0[0-2]$} & & & \\
\hline Hommes & \multicolumn{2}{|c|}{$2[0-4]$} & \multicolumn{2}{|c|}{$20[6-50]$} & & & \\
\hline \multicolumn{7}{|c|}{ Sexualité au cours des 12 derniers mois } & 0,000 \\
\hline Femmes uniquement & 1764 & 71,9 & 33 & 0,7 & 1797 & 24,5 & \\
\hline Hommes uniquement & 223 & 9,1 & 4264 & 87,6 & 4487 & 61,3 & \\
\hline Femmes et hommes & 205 & 8,3 & 191 & 3,9 & 396 & 5,4 & \\
\hline Non & 263 & 10,7 & 382 & 7,8 & 645 & 8,8 & \\
\hline Total & 2455 & 100,0 & 4870 & 100,0 & 7325 & 100,0 & \\
\hline \multicolumn{3}{|c|}{ Nombre médian de partenaires (12 derniers mois) } & & & & & \\
\hline Femmes & \multicolumn{2}{|c|}{$1[1-2]$} & \multicolumn{2}{|c|}{$0[0-0]$} & & & \\
\hline
\end{tabular}

${ }^{40}$ N. Bajos et N. Beltzer, «Les sexualités homo-bisexuelles : d'une acceptation de principe aux vulnérabilités sociales et préventives ", in N. BAJos et M. Bozon (éd.), Enquête sur la sexualité en France : Pratiques, genre et santé, Paris, La Découverte, 2008, p. 243-271. 


\begin{tabular}{|l|r|r|r|r|r|r|r|}
\hline Hommes & \multicolumn{2}{|c|}{$0[0-0]$} & \multicolumn{2}{|c|}{$4[1-10]$} & & & \\
\hline Statut matrimonial & & & & & & & 0,000 \\
\hline Célibataire & 2119 & 82,7 & 4432 & 88,4 & 6551 & 86,4 & \\
\hline PAcsé & 353 & 13,8 & 494 & 9,9 & 847 & 11,2 & \\
\hline Autre & 91 & 3,5 & 89 & 1,7 & 180 & 2,4 & \\
\hline Total & 2563 & 100,0 & 5015 & 100,0 & 7578 & 100,0 & \\
\hline Relation stable même sexe (12 derniers mois) & 746 & 30,4 & 1876 & 38,5 & 2622 & 35,8 & \\
\hline Non & 709 & 69,6 & 2994 & 61,5 & 4703 & 64,2 & \\
\hline Oui & 1709 & & & & & 0,000 \\
\hline Total & 2455 & 100,0 & 4870 & 100,0 & 7325 & 100,0 & \\
\hline
\end{tabular}

Lecture : $13,8 \%$ des lesbiennes et $9,9 \%$ des gays âgés de 15 à 35 ans ont déclaré être Pacsés avec une personne de même sexe.

Source : Enquête Presse Gays et Lesbiennes 2011.

\section{Parcours homosexuels : des sociabilités qui se distinguent en fonction des rapports de genre}

\section{Lieux de convivialité homosexuels et variabilité lesbiennes et gays}

En tant que lien identitaire et pourvoyeurs de « normes » culturelles et préventives, les lieux de convivialités homosexuels sont des vecteurs de socialisation et de liberté des minorités sexuelles. Ces espaces sont-ils investis de la même manière par les lesbiennes et les gays?

D'emblée, leur fréquentation varie selon la finalité des lieux. Ainsi les lieux purement conviviaux comme les bars ou les clubs sont plus fréquentés par les lesbiennes que par les gays. Dans ces espaces collectifs de visibilité et d'affirmation de l'homosexualité, «l'entre-soi » est de mise ${ }^{41}$. Les répondant.e.s qui se définissent comme homosexuel.le.s déclarent plus souvent fréquenter les bars ou clubs que ceux et celles qui se définissent comme bisexuel.le.s ou hétérosexuel.le.s, et ce de manière plus marquée pour les FSF. Quel que soit le sexe des répondants, la fréquentation augmente avec l'âge. Par ailleurs, les marqueurs sociaux influent sur la fréquentation de ces lieux : plus les répondants appartiennent à un milieu favorisé et plus ils/elles fréquentent ces espaces, et cela plus systématiquement chez les gays que chez les lesbiennes.

Ce constat traduit des inégalités dans l'usage de l'espace public liées à une histoire de l'homosexualité différente que l'on soit gay ou lesbienne ${ }^{42}$, mais aussi des interactions genrées à dominante masculine. Comme le montre l'ensemble des études françaises et anglo-saxonnes, portant sur le lien entre sexualités et territoires ${ }^{43}$,

41 D. Eribon, Réflexions sur la question gay, Paris, Flammarion, 2012 [1999], p. 210.

42 L. Chamberland, B. Franck et al. (éd.), Minorités sexuelles et constructions des genres, Sainte-Foy, Presses de l’Université du Québec, 2009.

43 J.-A. Podmore et A. Julie, "Gone « underground »? Lesbian visibility and the consolidation of queer space in Montreal », Social \& Cultural Geography, 7/4, 2006, p. 595 625 ; M. BLidon, Distance et rencontre. Eléments pour une géographie des homosexualités, thèse de géographie, sous la dir. de Christian Grataloup, Université Paris 7 Denis-Diderot, 
les fondements de ces inégalités renforcent l'invisibilité territoriale lesbienne ${ }^{44}$. Par exemple, l'étude de Castells ${ }^{45}$, qui interroge la spatialité des gays et des lesbiennes à San Francisco, montre que les gays ont un comportement dans l'espace urbain, typiquement masculin, territorialisé et visible autour d'espaces commerciaux et de consommation, qui participent à la gentrification des villes, alors que les lesbiennes se conforment à un comportement « féminin » non territorial, fondé sur des réseaux informels ${ }^{46}$, et se montrent plus politisées que les gays. La plus faible présence de « territoires lesbiens » peut aussi s'expliquer en grande partie par les inégalités de salaires entre les femmes et les hommes.

\section{Usage des sites de rencontre : distinction de genre et de capital social}

Les sites de rencontres sur internet sont peu fréquentés par les lesbiennes : la moitié ne les fréquente jamais contre $16 \%$ pour les gays, et seules $13 \%$ les fréquentent régulièrement contre la moitié pour les gays. Cette désaffection des sites par les FSF s'accentue avec l'âge, ce qui rend compte d'un effet de génération. De même, la nonfréquentation de ces espaces de rencontres virtuelles est d'autant plus importante chez les FSF qu'elles appartiennent à un milieu socio-culturel élevé. Ce résultat quantitatif complète les données qualitatives de l'enquête portant sur l'usage numérique des lesbiennes de Natacha Chetcuti-Osorovitz ${ }^{47}$. Elle montre que si l'échange numérique peut fonder les premières socialisations amicales et affectives lesbiennes, il relève d'un capital culturel spécifique ou d'une sociabilité homosexuelle moins importante. Les jeunes lesbiennes qui font des études supérieures vont davantage s'engager dans l'association LGBT de leur université, d'autres s'orienteront vers les bars lesbiens et gays de leur ville. Pour ces dernières, les pratiques de sociabilité passent par des réseaux associatifs ou des réseaux commerciaux existants. Toutefois, l'âge, le capital scolaire ou la classe sociale ont moins d'incidences sur l'usage du Net en début de parcours sexuel que la situation géographique, qui peut, dans certains cas, être un facteur plus important.

A l'inverse, les gays, quel que soient leur capital social ou culturel, fréquentent très largement ces sites $(75 \%)$, la moitié de façon régulière. La fréquentation augmente avec l'âge, mais est inversement proportionnelle au niveau d'étude ou au milieu social. Ces observations corroborent les travaux de Marie Bergström sur la topographie des sites de rencontres : les sites lesbiens sont peu nombreux et valorisent « la mise en relation avec un partenaire stable », alors que les sites de rencontres gays proposent une multiplicité de scénarios sexuels ${ }^{48}$.

2007 ; A. Alessandrin et Y. Raibaud, Géographie des homophobies, Paris, Armand Colin, 2013.

${ }^{44}$ N. Cattan et S. Leroy, «La ville négociée : les homosexuel(le)s dans l'espace public parisien », Cahiers de géographie du Québec, 54/151, 2010, p. 9-24.

${ }^{45}$ M. CASTElls, The Rise of the Network Society, The Information Age : Economy, Society and Culture, Cambridge, (Mass.), Oxford, Blackwell, 1996.

${ }^{46}$ M. Perrot, Histoire de chambres, Paris, Le Seuil, 2009.

47 N. Chetcuti-Osorovitz, "Sexualités entre femmes et usage numérique », op. cit.

${ }^{48}$ M. Bergström, « La toile des sites de rencontre en France. Topographie d'un nouvel espace social en ligne », Réseaux, 2/166, 2011, p. 225-260. 


\section{Le cercle amical}

L'entourage amical est pour près de la moitié des répondants.es majoritairement hétérosexuel. Le " repli " sur un réseau exclusivement homosexuel est un comportement minoritaire aussi bien pour les FSF que pour les HSH de ce corpus. Au fil du temps, l'entourage amical se modifie. Ainsi, avec l'âge, à partir d'une socialisation majoritairement hétérosexuelle, les FSF et les HSH recherchent un entourage plus compréhensif, voire moins violent vis-à-vis de leur orientation sexuelle et intègrent des cercles amicaux plus mixtes. La fréquentation des pairs joue un rôle important de socialisation et de soutien, en particulier durant les phases de dévoilement de l'orientation sexuelle, susceptibles de générer des périodes de stress importantes. Les jeunes homosexuel.le.s doivent, au cours d'une même période, prendre conscience de leurs préférences sexuelles, les assumer et affronter le regard de l'autre ${ }^{49}$. Cependant, le réseau amical hétérosexuel des répondants.es fait preuve d'une ouverture d'esprit puisque, plus de $80 \%$ de leurs ami.e.s connaissent leur orientation sexuelle.

Tableau 3. Sociabilité des lesbiennes et des gays ayant répondu à l'EPGL 2011 âgés de 15 à 35 ans

\begin{tabular}{|l|r|r|r|r|r|r|c|}
\hline \multirow{2}{*}{} & \multicolumn{2}{|c|}{ Femmes } & \multicolumn{2}{|c|}{ Hommes } & \multicolumn{2}{c|}{ Total } & Ecart F-H \\
\cline { 2 - 8 } & Effectifs & $\%$ & Effectifs & $\%$ & Effectifs & $\%$ & p-value \\
\hline Fréquentation des bars ou clubs sans backroom & & & & & 0,000 \\
$\quad$ Jamais & 688 & 26,6 & 1682 & 34,5 & 2370 & 31,7 & \\
Occasionnellement & 1452 & 56,1 & 2309 & 47,4 & 3761 & 50,4 & \\
$\quad$ Régulièrement & 450 & 17,3 & 885 & 18,1 & 1335 & 17,9 & \\
Total & 2590 & 100,0 & 4876 & 100,0 & 7466 & 100,0 & \\
\hline Fréquentation des sites de rencontre sur Internet & & & & 0,000 \\
$\quad$ Jamais & 1343 & 53,2 & 792 & 15,9 & 2135 & 28,5 & \\
$\quad$ Occasionnellement & 853 & 33,7 & 1666 & 33,5 & 2519 & 33,6 & \\
$\quad$ Régulièrement & 332 & 13,1 & 2512 & 50,6 & 2844 & 37,9 & \\
Total & 2528 & 100,0 & 4970 & 100,0 & 7498 & 100,0 & \\
\hline Réseau d'amitiés & & & & & & & 0,000 \\
$\quad$ Majoritairement homosexuels & 298 & 11,4 & 624 & 12,5 & 922 & 12,1 & \\
$\quad$ Majoritairement hétérosexuels & 1240 & 47,7 & 2541 & 50,7 & 3781 & 49,7 & \\
$\quad \begin{array}{l}\text { Parmi eux, autant d'homo- } \\
\text { sexuels que d'hétérosexuels }\end{array}$ & 1043 & 40,1 & 1740 & 34,8 & 2783 & 36,6 & \\
$\quad$ Vous n'avez pas d'ami & 22 & 0,8 & 100 & 2,0 & 122 & 1,6 & \\
Total & 2603 & 100,0 & 5005 & 100,0 & 7608 & 100,0 & \\
\hline
\end{tabular}

Lecture : $53,1 \%$ des lesbiennes et $15,9 \%$ des gays âgés de 15 à 35 ans ont déclaré ne jamais fréquenter les sites de rencontre sur internet.

Source : Enquête Presse Gays et Lesbiennes 2011.

${ }^{49}$ A. Velter, A. Bouyssou-Michel et al., Enquête Presse Gay 2004 Saint-Maurice, op. cit. 


\section{Connaissance de l'homosexualité par les parents et variation selon le milieu social d'origine}

Le dévoilement de son homosexualité - auprès des proches et/ou publiquement est devenu constitutif des processus identitaires gays et lesbiens de la fin du $\mathrm{XX}^{\mathrm{e}}$ siècle. Annoncer son orientation sexuelle est loin d'être aisé et expose à des actes de rejet ; même si la société semble plus tolérante que par le passé à l'égard des homosexuels, il s'agit encore d'une acceptation sociale de principe ${ }^{50}$. Le sentiment d'isolement peut être accentué par des actes de rejet. Ces événements concomitants peuvent entraîner des atteintes importantes à l'estime de soi ${ }^{51}$.

La perception, la connaissance et l'acceptation de son orientation sexuelle par soi et par ses parents sont plus élevées chez les FSF que chez les HSH avec un écart positif pour les mères.

Les FSF sont $67 \%$ à indiquer que leur père connaît leur orientation sexuelle contre $62 \%$ pour les HSH. Les mères semblent plus au fait de la vie intime de leurs enfants.

Avec l'âge, que l'on soit une femme ou un homme, la perception de la connaissance de son orientation sexuelle par ses parents augmente, mais à des niveaux et des rythmes différents. A 15-19 ans, 44\% des répondant.e.s indiquent que leur père connaît leur orientation sexuelle, alors que la connaissance des mères est déjà de l'ordre de $60 \%$ pour les jeunes filles et $56 \%$ pour les jeunes garçons $(\mathrm{p}<0,000)$. Mais après 20 ans, des écarts sont observés entre les FSF et les HSH en défaveur des hommes : à 31-35 ans, $85 \%$ des FSF déclarent que leur orientation sexuelle est connue par leur père pour $70 \%$ des HSH. L'écart est moindre pour les mères à ces âges : $88 \%$ pour les FSF et $80 \%$ pour les HSH.

La perception de la connaissance de l'orientation sexuelle pour les FSF par leur père et leur mère augmente avec leur niveau d'étude (pour le père, on passe de $58 \%$ pour celles qui n'ont pas suivi d'études supérieures à $69 \%$ pour celles qui en ont suivi) et ce, quel que soit leur âge, de même pour les HSH avec leur mère. En revanche, après stratification sur l'âge, il n'est pas constaté de différences significatives selon le niveau de diplôme concernant la connaissance de l'orientation des HSH par leur père.

Le fait que les pères soient systématiquement moins au courant de l'orientation sexuelle de leurs enfants peut s'expliquer par plusieurs éléments. D'une part, les mères semblent continuer à être les interlocutrices privilégiées de leurs enfants sur les questions intimes comme l'observent Michel Bozon ${ }^{52}$ concernant les demandes d'information sur la contraception ou encore Geneviève Cresson sur le rôle d'agent sanitaire des mères ${ }^{53}$. D'autre part, on peut faire l'hypothèse d'une intériorisation plus ou moins consciente et sans doute plus prégnante chez les HSH que chez les FSF, de la moins bonne acceptation de l'homosexualité par les hommes que par les femmes.

50 N. Bajos et N. Beltzer., « Les sexualités homo-bisexuelles », op. cit.

51 L.-M. Paget, Ch. Chan Chee et al., « Facteurs associés aux tentatives de suicide chez les minorités sexuelles : résultats de l'enquête presse gays et lesbiennes 2011 », Revue d'épidémiologie et de santé publique, 64/3, 2015, p. 153-163.

52 M. Bozon, « Premier rapport sexuel, première relation », op. cit.

53 G. CRESSON, «Les soins profanes et la division du travail entre hommes et femmes », in P. AÏACH, D. CÈBE et al. (éd.), Femmes et hommes dans le champ de la santé. Approches sociologiques, Rennes, ENSP, 2001, p. 303-328. 
En effet, l'enquête CSF avait montré que les hommes déclaraient qu'ils auraient plus de mal à accepter sans difficulté l'homosexualité de leurs enfants que les femmes ${ }^{54}$.

La connaissance de l'orientation sexuelle par le père et la mère est moindre pour les HSH issus de milieux populaires que pour ceux issus de milieux favorisés, quel que soit leur âge, ce qui n'est pas le cas pour les FSF.

Le lieu de résidence est discriminant aussi. Quel que soit le sexe des répondants, celles et ceux qui habitent dans une agglomération urbanisée de plus de 100000 habitants déclarent que leurs parents sont plus au courant de leur homosexualité que ceux habitant dans une petite commune et cela après stratification sur l'âge, le niveau d'étude et l'origine sociale.

Tableau 4. Connaissance et acceptation du père et de la mère de l'orientation sexuelle des lesbiennes et des gays ayant répondu à l'EPGL 2011 âgés de 15 à 35 ans

\begin{tabular}{|l|r|r|r|r|r|r|r|}
\hline & \multicolumn{2}{|c|}{ Femmes } & \multicolumn{2}{|c|}{ Hommes } & \multicolumn{2}{|c|}{ Total } & \multirow{2}{*}{ Ecart F-H } \\
\hline & Effectifs & $\%$ & Effectifs & $\%$ & Effectifs & $\%$ & p-value \\
\hline Connaissance par le père de l'orientation sexuelle (pop. concernée) & & & 0,000 \\
\hline Non & 754 & 33,3 & 1730 & 38,4 & 2484 & 36,7 & \\
\hline Oui & 1510 & 66,7 & 2777 & 61,6 & 4287 & 63,3 & \\
\hline Total & 2264 & 100,0 & 4507 & 100,0 & 6771 & 100,0 & \\
\hline Acceptation de l'orientation sexuelle par le père (si connue) \\
\hline Acceptée & 1197 & 79,4 & 2133 & 77,6 & 3330 & 78,2 & \\
\hline Rejetée & 99 & 6,6 & 262 & 9,5 & 361 & 8,5 & \\
\hline Vous ne savez pas & 211 & 14,0 & 354 & 12,9 & 565 & 13,3 & \\
\hline Total & 1507 & 100,0 & 2749 & 100,0 & 4256 & 100,0 & \\
\hline Connaissance par la mère de l'orientation sexuelle (pop. concernée) & & 0,000 \\
\hline Non & 598 & 24,3 & 1347 & 28,0 & 1945 & 26,7 & \\
\hline Oui & 1864 & 75,7 & 3467 & 72,0 & 5331 & 73,3 & \\
\hline Total & 2462 & 100,0 & 4814 & 100,0 & 7276 & 100,0 & \\
\hline Acceptation de l'orientation sexuelle par la mère (si connue) \\
\hline Acceptée & 1480 & 79,5 & 2977 & 86,6 & 4457 & 84,1 & \\
\hline Rejetée & 169 & 9,1 & 207 & 6,0 & 376 & 7,1 & \\
\hline Vous ne savez pas & 212 & 11,4 & 252 & 7,4 & 464 & 8,8 & \\
\hline Total & 1861 & 100,0 & 3436 & 100,0 & 5297 & 100,0 & \\
\hline
\end{tabular}

Lecture : $66,7 \%$ des lesbiennes et $61,6 \%$ des gays âgés de 15 à 35 ans ont déclaré que leur père connaissait leur orientation sexuelle.

Source : Enquête Presse Gays et Lesbiennes 2011.

Le fait de résider chez ses parents, quel que soit son sexe ou son âge, a un impact sur la connaissance ou pas de l'orientation sexuelle. Ainsi, parmi les répondants vivant

\footnotetext{
${ }^{54}$ N. Bajos et N. Beltzer, « Les sexualités homo-bisexuelles », op. cit.
} 
dans le foyer parental, $48 \%$ indiquent que leur père connait leur orientation sexuelle contre $67 \%$ parmi ceux ayant quitté ce foyer (mise à distance du réseau de parenté). La mise à distance du réseau de parenté joue en faveur d'une plus grande connaissance des parents, mais avec des gradients selon le sexe des répondants et selon qu'ils sont célibataires ou vivent en couple. Ainsi, les lesbiennes étant plus souvent dans des formes conjugales que les gays au multipartenariat sexuel plus important, on peut supposer que l'intégration de l'homosexualité est plus forte chez les lesbiennes auprès de leur parent, car elles passent par des relations électives hétéronormées et non par la catégorisation sexuelle. Il est plus facile de se faire reconnaître en tant que couple, qu'en tant que gay ou lesbienne sans partenaire stable.

Bien que les niveaux d'acceptation de l'orientation sexuelle des répondants.e.s soient élevés dans notre population d'étude, des différences de genre sont observées selon qu'il s'agit du père ou de la mère. L'orientation sexuelle des FSF est mieux acceptée que celle des HSH par les pères. En revanche, les FSF rapportent une moindre acceptation de leur orientation sexuelle par leur mère que les HSH. Il faut noter qu'un décalage entre connaissance et acceptation existe : être au courant ne signifie pas accepter. Ainsi, 13\% des répondants.es ne savent pas si leur homosexualité est acceptée par leur père contre $9 \%$ pour les mères alors que leur orientation sexuelle est connue. Cette méconnaissance des mères est plus importante pour les lesbiennes que pour les gays. Concernant l'acceptation, on retrouve les mêmes dichotomies sexuées des parents. De manière significative, les mères acceptent plus que les pères l'homosexualité de leurs enfants ( $84 \%$ vs $78 \%$ ). Elles acceptent plus l'homosexualité de leurs garçons que celle de leurs filles ( $87 \%$ vs $79 \%)$, alors que les pères acceptent de la même manière l'homosexualité de leur enfant quel que soit leur sexe (79\% vs $77 \%$, NS). Indépendamment du sexe des répondants.es, l'acceptation de leur homosexualité par leurs parents augmente avec l'âge.

Alors que comme nous l'avons noté plus haut, l'homosexualité semble mieux acceptée dans la population générale qu'auparavant, des spécificités méritent d'être évoquées. Tout d'abord des différences générationnelles : l'acceptation des personnes plus âgées susceptibles d'être les parents de jeunes adultes des mêmes classes d'âge que les répondants.es de notre étude reste problématique. Par ailleurs, il n'est pas observé de différence d'acceptation de l'homosexualité de leur fils ou fille de la part des pères selon plusieurs marqueurs sociaux, que ce soit le niveau d'études des répondants.es ou leur origine sociale. Pour les mères, l'acceptation de l'homosexualité, uniquement, de leurs filles, est plus importante parmi les répondants.es de milieu social intermédiaire (83\%) mais n'est pas différente pour les classes aisées (79\%) ou populaires $(75 \%)$ après ajustement sur l'âge. Ces résultats corroborent les thèses de Wilfried Rault ${ }^{55}$, sur le présupposé d'une meilleure acceptation par les classes supérieures que par les autres classes sociales, qui en fait ne serait qu'une « tolérance de principe » mais moins prégnante dans la pratique. Ainsi, alors que les répondants.es d'origine sociale

55 W. RAult, « Les attitudes « gayfriendly » en France : entre appartenances sociales, trajectoires familiales et biographies sexuelles », Actes de la recherche en sciences sociales, 213/3, 2016, p. 38-65. 
aisée indiquaient une meilleure connaissance de leur homosexualité par rapport aux autres classes, cette différence disparaît pour ce qui concerne l'acceptation.

Cet article s'est intéressé aux premières socialisations des jeunes lesbiennes et gays sous le prisme des rapports de genre. Plusieurs indicateurs ont été mobilisés pour démontrer, d'une part, l'existence d'un rapport de pouvoir asymétrique et systématique entre les hommes gay et les femmes lesbiennes et, d'autre part, la persistance d'une contrainte à la norme hétérosexuelle produisant des modes d'identification et de socialisation différenciés selon que l'on est gay ou lesbienne.

A partir d'un corpus qui se caractérise par des répondants.es diplômé.e.s, appartenant à des catégories socio-professionnelles privilégiées et résidant en zone urbaine, des inégalités sociales de genre sont constatées en termes de rapport entre l'emploi, le diplôme et le revenu, rendant compte d'une hiérarchisation des sexes au sein des minorités sexuelles. Ainsi, si dans la présente enquête, les lesbiennes appartiennent à un milieu social plus favorisé que les gays et si elles sont plus diplômées que leurs homologues, elles occupent des professions moins prestigieuses et moins en adéquation avec leur niveau d'étude et ont de fait un moindre niveau de revenu.

Les dynamiques de socialisation des jeunes lesbiennes et gays sont marquées par une contrainte à la norme hétérosexuelle qui se manifeste différemment selon la position de genre. Cette pression normative est appréhendée et contournée de manière dissemblable. Ainsi, les jeunes lesbiennes semblent avoir intériorisé les normes de reproduction sexuelle liée à leur position de genre en se définissant plus souvent comme bisexuelles, en commençant leur sexualité plus souvent avec un homme, en ayant moins de partenaires sexuels quel que soit leur sexe. Elles privilégient des relations amoureuses monopartenaires, des relations amicales électives. Leur orientation sexuelle est, plus que pour les gays, connue et acceptée par leurs parents. Si le lesbianisme semble davantage intégré dans les cercles familiaux que l'homosexualité masculine, c'est qu'elle renvoie à un imaginaire désexualisé et temporaire. L'invisibilité sociale du lesbianisme participe de cette hétéronormativité.

Les jeunes gays, quant à eux, se distancient des normes hétérosexuelles en mettant en œuvre des stratégies d'échappement par « une mobilité sociale ascendante » afin de s'en affranchir comme le faisaient déjà les générations précédentes. Rapidement, ils s'approprient les nouveaux codes de socialisation propre à l'homosexualité masculine conciliant sphère sociale affective et sexuelle. Ces jeunes gays se définissent plus souvent comme homosexuels, décrivent un parcours sexuel plus systématiquement ou rapidement uniquement avec des hommes et un nombre de partenaires sexuels important ; leur orientation sexuelle est moins connue et acceptée par la famille. Aussi, bien que se démarquant de la norme hétérosexuelle, les jeunes gays comme leurs aînés se conforment aux images traditionnelles des masculinités confortées par un modèle de virilité hétéronormé. 